PROCEEDINGS OF THE

AMERICAN MATHEMATICAL SOCIETY

Volume 126, Number 6, June 1998, Pages 1661-1667

S 0002-9939(98)04503-1

\title{
ON THE OSCILLATION OF NONLINEAR TWO-DIMENSIONAL DIFFERENTIAL SYSTEMS
}

\author{
I.-G. E. KORDONIS AND CH. G. PHILOS
}

(Communicated by Hal L. Smith)

\begin{abstract}
An oscillation criterion is given for a certain form of nonlinear two-dimensional differential systems. This criterion originated in a well-known oscillation result due to Coles (as extended and improved by Wong) concerning second order nonlinear differential equations with alternating coefficients.
\end{abstract}

\section{INTRODUCTION AND STATEMENT OF THE MAIN RESULT}

The oscillation problem for second order nonlinear differential equations is of particular interest and, therefore, it is the subject of many investigations. It is an interesting problem to extend oscillation criteria for second order nonlinear differential equations to the case of nonlinear two-dimensional differential systems. Such differential systems include in particular the second order nonlinear differential equations. In this paper, a classical oscillation criterion due to Coles [3] (as extended and improved by Wong [11], [12]) for second order nonlinear differential equations is extended to nonlinear two-dimensional differential systems.

Consider the nonlinear two-dimensional differential system

$$
x^{\prime}(t)=b(t) g[y(t)], y^{\prime}(t)=-a(t) f[x(t)],
$$

where $a$ and $b$ are continuous real-valued functions on an interval $\left[t_{0}, \infty\right)$, and $f$ and $g$ are continuous real-valued functions on the real line $\mathbb{R}$ with the sign property

$$
u f(u)>0 \text { and } u g(u)>0 \text { for all } u \in \mathbb{R}-\{0\} .
$$

It will be supposed that $b$ is nonnegative on $\left[t_{0}, \infty\right), f$ is continuously differentiable on $\mathbb{R}-\{0\}$ and satisfies

$$
f^{\prime}(u) \geq 0 \text { for every } u \neq 0,
$$

and $g$ is increasing on $\mathbb{R}$. Note that no restriction is imposed on the sign of the coefficient $a$.

Throughout the paper, we shall restrict our attention only to the solutions of the differential system $(\mathrm{S})$ which exist on some ray $\left[T_{0}, \infty\right)$, where $T_{0} \geq t_{0}$ may depend on the particular solutions. Note that under quite general conditions there will always exist solutions of $(\mathrm{S})$ which are continuable to an interval $\left[T_{0}, \infty\right), T_{0} \geq t_{0}$, even though there will also exist noncontinuable solutions.

Received by the editors September 23, 1996.

1991 Mathematics Subject Classification. Primary 34C10.

Key words and phrases. Differential system, solution, oscillation.

(C)1998 American Mathematical Society 
As usual, a continuous real-valued function defined on an interval $\left[T_{0}, \infty\right)$ is said to be oscillatory if it has arbitrarily large zeros, and otherwise it is said to be nonoscillatory. A solution $(x, y)$ of the differential system (S) will be called oscillatory if both $x$ and $y$ are oscillatory functions, and otherwise it will be called nonoscillatory. The differential system $(\mathrm{S})$ will be called oscillatory if all its solutions are oscillatory.

It is remarkable that, in the case where the coefficient $b$ is assumed to be not identically zero on any interval of the form $\left[\tau_{0}, \infty\right), \tau_{0} \geq t_{0}$, from the first equation of (S) it follows easily that, for any solution $(x, y)$ of the differential system (S), the oscillation of $x$ implies that $y$ is also oscillatory. So, in this case, if $(x, y)$ is a nonoscillatory solution of (S), then $x$ is always nonoscillatory.

Some oscillation results for the differential system (S) have been presented by Kwong and Wong [5]. More precisely, generalizations of Atkinson's theorem [1] for second order superlinear differential equations and of Belohorec's theorem [2] for second order sublinear equations are given in [5]. Moreover, in [5] an extension of Waltman's classical theorem [9] for second order nonlinear differential equations is established.

The special case where $f(u)=|u|^{\lambda} \operatorname{sgn} u, u \in \mathbb{R}$ and $g(u)=|u|^{\mu} \operatorname{sgn} u, u \in \mathbb{R}(\lambda>$ $0, \mu>0)$ is of particular interest. In this case, the differential system (S) becomes

$$
x^{\prime}(t)=b(t)|y(t)|^{\mu} \operatorname{sgn} y(t), y^{\prime}(t)=-a(t)|x(t)|^{\lambda} \operatorname{sgn} x(t),
$$

where $\lambda$ and $\mu$ are positive constants. System $\left(\mathrm{S}_{0}\right)$ is the prototype of $(\mathrm{S})$. For some oscillation results for the differential system $\left(\mathrm{S}_{0}\right)$ we refer to Mirzov [6],[7], [8].

In the particular case where $b$ is positive on $\left[t_{0}, \infty\right)$ and $g(u)=u, u \in \mathbb{R}$, the differential system $(\mathrm{S})$ reduces to the second order nonlinear differential equation

$$
\left[x^{\prime}(t) / b(t)\right]^{\prime}+a(t) f[x(t)]=0 .
$$

For $b(t)=1$ for $t \geq t_{0}$, the last equation becomes

$$
x^{\prime \prime}(t)+a(t) f[x(t)]=0 .
$$

The prototype of $(\mathrm{E})$ is the differential equation

$$
x^{\prime \prime}(t)+a(t)|x(t)|^{\lambda} \operatorname{sgn} x(t)=0,
$$

where $\lambda>0$. System $\left(\mathrm{S}_{0}\right)$ is reduced to $\left(\mathrm{E}_{0}\right)$ when $b(t)=1$ for $t \geq t_{0}$ and $\mu=1$.

Kwong and Wong [5] established that, if the coefficient $b$ is such that

$$
\int_{t_{0}}^{\infty} b(t) d t=\infty
$$

and $g$ satisfies

$$
\liminf _{u \rightarrow \pm \infty}|g(u)|>0
$$

then the condition

$$
\int_{t_{0}}^{\infty} a(s) d s=\infty
$$

is sufficient for the oscillation of the differential system (S). [Note that this result holds true without the assumption that $g$ is increasing on $\mathbb{R}$. Clearly, the increasing character of $g$ on $\mathbb{R}$ implies condition $\left(\mathrm{C}_{2}\right)$.] This oscillation criterion is an extension of a well-known oscillation theorem due to Waltman [9] (see also Wong [10] and 
Kwong and Wong [4]) for second order nonlinear differential equations with alternating coefficients. Our purpose here is to examine the oscillation of the differential system $(\mathrm{S})$ in a case where $\left(\mathrm{C}_{3}\right)$ fails. More precisely, our interest is concentrated to the case where

$$
\int_{t_{0}}^{\infty} a(s) d s \text { exists as a real number. }
$$

Throughout the paper, if $\left(\mathrm{C}_{4}\right)$ holds, by $A$ we will denote the function defined by

$$
A(t)=\int_{t}^{\infty} a(s) d s, t \geq t_{0} .
$$

Coles [3] showed that, if $\left(\mathrm{C}_{4}\right)$ holds and $A(t) \geq 0$ for $t \geq t_{0}$, then the condition

$$
\int_{t_{0}}^{\infty} A(t) d t=\infty
$$

suffices for the oscillation of the differential equation $\left(\mathrm{E}_{0}\right)$ in the superlinear case $\lambda>1$. This result has been improved by Wong [11], who proved that the assumption that $A$ is nonnegative on $\left[t_{0}, \infty\right)$ can be removed. In [12], Wong extended Cole's criterion (without the restriction that $A(t) \geq 0$ for $t \geq t_{0}$ ) for the more general case of the differential equation (E), where $f$ is strongly superlinear in the sense that

$$
\int^{\infty} \frac{d u}{f(u)}<\infty \text { and } \int^{-\infty} \frac{d u}{f(u)}<\infty .
$$

More precisely, Wong [12] established the following oscillation theorem for the differential equation $(\mathrm{E})$.

Theorem 0. Let $(\mathrm{E})$ be strongly superlinear in the sense that $\left(\mathrm{C}_{6}\right)$ is satisfied, and suppose that $\left(\mathrm{C}_{4}\right)$ holds. Then $\left(\mathrm{C}_{5}\right)$ is a sufficient condition for the oscillation of the differential equation $(\mathrm{E})$.

Our aim in this paper is to present an extension of Theorem 0 to the differential system (S). For this purpose, we introduce the following assumption:

$\left(\mathrm{C}_{7}\right)$ For all sufficiently small $u$ and every $v>0$

$$
g(u) g(v) \leq g(u v) \leq g(u)[-g(-v)] .
$$

This assumption holds by itself in the case where $g(u)=|u|^{\mu} \operatorname{sgn} u, u \in \mathbb{R}(\mu>0)$, and especially when $g(u)=u, u \in \mathbb{R}$. We will consider the case where the function $g \circ f$ is strongly superlinear in the sense that

$$
\int^{\infty} \frac{d u}{g[f(u)]}<\infty \text { and } \int^{-\infty} \frac{d u}{g[f(u)]}<\infty .
$$

Note that in the special case where $g(u)=u, u \in \mathbb{R}$, condition $\left(\mathrm{C}_{8}\right)$ is reduced to $\left(\mathrm{C}_{6}\right)$.

Our main result is the following theorem.

Theorem. Let the function $g \circ f$ be strongly superlinear in the sense that $\left(\mathrm{C}_{8}\right)$ is satisfied, and suppose that $\left(\mathrm{C}_{1}\right),\left(\mathrm{C}_{4}\right)$ and $\left(\mathrm{C}_{7}\right)$ hold. The differential system $(\mathrm{S})$ is oscillatory if

$$
\int_{t_{0}}^{\infty} b(t) g[A(t)] d t=\infty .
$$

Our theorem reduces to Theorem 0 in the special case $b(t)=1$ for $t \geq t_{0}$ and $g(u)=u, u \in \mathbb{R}$. 


\section{A TECHNICAL LEMMA}

In order to prove our theorem we make use of the following technical lemma.

Lemma. Let $(x, y)$ be a solution on an interval $[\tau, \infty), \tau \geq t_{0}$, of the differential system $(\mathrm{S})$ with $x(t)>0$ for all $t \geq \tau$. Moreover, let $\tau^{\star} \geq \tau$ and $c$ be a real constant. If

$$
-\frac{y(\tau)}{f[x(\tau)]}+\int_{\tau}^{t} a(s) d s+\int_{\tau}^{\tau^{\star}} \frac{y(s) x^{\prime}(s) f^{\prime}[x(s)]}{\{f[x(s)]\}^{2}} d s \geq c \text { for every } t \geq \tau^{\star},
$$

then

$$
y(t) \leq-c f\left[x\left(\tau^{\star}\right)\right] \text { for all } t \geq \tau^{\star} .
$$

Proof. From the second equation of (S) we obtain for $t \geq \tau^{\star}$

$$
\int_{\tau}^{t} a(s) d s=\int_{\tau}^{t} \frac{-y^{\prime}(s)}{f[x(s)]} d s=-\frac{y(t)}{f[x(t)]}+\frac{y(\tau)}{f[x(\tau)]}-\int_{\tau}^{t} \frac{y(s) x^{\prime}(s) f^{\prime}[x(s)]}{\{f[x(s)]\}^{2}} d s
$$

and so we have

$$
\begin{aligned}
-\frac{y(t)}{f[x(t)]}= & {\left[-\frac{y(\tau)}{f[x(\tau)]}+\int_{\tau}^{t} a(s) d s+\int_{\tau}^{\tau^{\star}} \frac{y(s) x^{\prime}(s) f^{\prime}[x(s)]}{\{f[x(s)]\}^{2}} d s\right] } \\
& +\int_{\tau^{\star}}^{t} \frac{y(s) x^{\prime}(s) f^{\prime}[x(s)]}{\{f[x(s)]\}^{2}} d s
\end{aligned}
$$

for every $t \geq \tau^{\star}$. This, by our hypothesis, gives

$$
-\frac{y(t)}{f[x(t)]} \geq c+\int_{\tau^{\star}}^{t}\left\{-\frac{y(s)}{f[x(s)]}\right\}\left\{-\frac{x^{\prime}(s) f^{\prime}[x(s)]}{f[x(s)]}\right\} d s \quad \text { for all } t \geq \tau^{\star} .
$$

Hence, by using a simple result from the theory of integral inequalities (see, for example, Kwong and Wong [4], Lemma 1), we conclude that

$$
w(t) \leq-y(t) \text { for every } t \geq \tau^{\star}
$$

where $w$ satisfies

$$
\frac{w(t)}{f[x(t)]}=c+\int_{\tau^{\star}}^{t} \frac{w(s)}{f[x(s)]}\left\{-\frac{x^{\prime}(s) f^{\prime}[x(s)]}{f[x(s)]}\right\} d s \text { for } t \geq \tau^{\star} .
$$

We can easily see that $w^{\prime}=0$ on $\left[\tau^{\star}, \infty\right)$. Moreover, we obviously have $w\left(\tau^{\star}\right)=$ $c f\left[x\left(\tau^{\star}\right)\right]$. Thus, $w(t)=c f\left[x\left(\tau^{\star}\right)\right]$ for all $t \geq \tau^{\star}$ and so the proof of the lemma is complete.

\section{Proof of the Theorem}

Assume that the differential system $(\mathrm{S})$ admits a nonoscillatory solution $(x, y)$ on an interval $\left[T_{0}, \infty\right)$, where $T_{0} \geq t_{0}$. From $\left(\mathrm{C}_{1}\right)$ it follows that the coefficient $b$ is not identically zero on any interval of the form $\left[\tau_{0}, \infty\right), \tau_{0} \geq t_{0}$. So, as pointed out in Section 1, $x$ is always nonoscillatory. Without loss of generality, we shall assume that $x(t) \neq 0$ for all $t \geq T_{0}$. Furthermore, we observe that the substitution $z=-x, w=-y$ transforms $(\mathrm{S})$ into the system

$$
z^{\prime}(t)=b(t) \widehat{g}[w(t)], w^{\prime}(t)=-a(t) \widehat{f}[z(t)],
$$

where $\widehat{f}(u)=-f(-u), u \in \mathbb{R}$, and $\widehat{g}(v)=-g(-v), v \in \mathbb{R}$. The functions $\widehat{f}$ and $\widehat{g}$ are subject to the conditions posed on $f$ and $g$. Thus, we can restrict our discussion only to the case where $x$ is positive on $\left[T_{0}, \infty\right)$. It must be noted that from the first 
equation of the system (S) it follows that the function $y x^{\prime}$ is necessarily nonnegative on the interval $\left[T_{0}, \infty\right)$, even though $y$ is oscillatory.

First of all, we will show that

$$
\int_{T_{0}}^{\infty} \frac{y(t) x^{\prime}(t) f^{\prime}[x(t)]}{\{f[x(t)]\}^{2}} d t<\infty
$$

To this end, let us assume that $(\star)$ fails. By condition $\left(\mathrm{C}_{4}\right)$, there exists a real constant $K$ such that

$$
-\frac{y\left(T_{0}\right)}{f\left[x\left(T_{0}\right)\right]}+\int_{T_{0}}^{t} a(s) d s \geq K \text { for } t \geq T_{0} .
$$

Furthermore, we can choose a point $T_{0}^{\star} \geq T_{0}$ so that

$$
\int_{T_{0}}^{T_{0}^{\star}} \frac{y(s) x^{\prime}(s) f^{\prime}[x(s)]}{\{f[x(s)]\}^{2}} d s \geq 1-K .
$$

So, we have

$$
-\frac{y\left(T_{0}\right)}{f\left[x\left(T_{0}\right)\right]}+\int_{T_{0}}^{t} a(s) d s+\int_{T_{0}}^{T_{0}^{\star}} \frac{y(s) x^{\prime}(s) f^{\prime}[x(s)]}{\{f[x(s)]\}^{2}} d s \geq 1 \text { for every } t \geq T_{0}^{\star}
$$

and hence, by applying our lemma with $\tau=T_{0}, \tau^{\star}=T_{0}^{\star}$ and $c=1$, we obtain

$$
y(t) \leq d \text { for all } t \geq T_{0}^{\star},
$$

where $d=-f\left[x\left(T_{0}^{\star}\right)\right]<0$. Next, from the first equation of (S) we derive for $t \geq T_{0}^{\star}$

$$
x(t)-x\left(T_{0}^{\star}\right)=\int_{T_{0}^{\star}}^{t} b(s) g[y(s)] d s \leq g(d) \int_{T_{0}^{\star}}^{t} b(s) d s,
$$

which, in view of $\left(\mathrm{C}_{1}\right)$, gives $\lim _{t \rightarrow \infty} x(t)=-\infty$, a contradiction.

Now, by taking into account $\left(\mathrm{C}_{4}\right)$ and the definition of the function $A$ as well as $(\star)$, from the second equation of (S) we get for $t \geq T_{0}$

$$
\begin{aligned}
A\left(T_{0}\right)-A(t)= & \int_{T_{0}}^{t} a(s) d s=\int_{T_{0}}^{t} \frac{-y^{\prime}(s)}{f[x(s)]} d s \\
= & -\frac{y(t)}{f[x(t)]}+\frac{y\left(T_{0}\right)}{f\left[x\left(T_{0}\right)\right]}-\int_{T_{0}}^{t} \frac{y(s) x^{\prime}(s) f^{\prime}[x(s)]}{\{f[x(s)]\}^{2}} d s \\
= & -\frac{y(t)}{f[x(t)]}+\frac{y\left(T_{0}\right)}{f\left[x\left(T_{0}\right)\right]}-\int_{T_{0}}^{\infty} \frac{y(s) x^{\prime}(s) f^{\prime}[x(s)]}{\{f[x(s)]\}^{2}} d s \\
& +\int_{t}^{\infty} \frac{y(s) x^{\prime}(s) f^{\prime}[x(s)]}{\{f[x(s)]\}^{2}} d s,
\end{aligned}
$$

namely

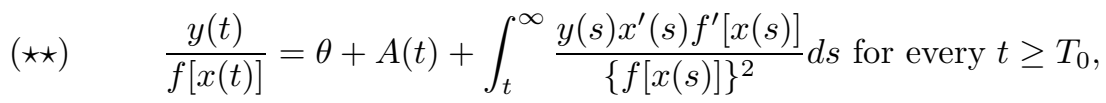

where the real number $\theta$ is defined by

$$
\theta=\frac{y\left(T_{0}\right)}{f\left[x\left(T_{0}\right)\right]}-A\left(T_{0}\right)-\int_{T_{0}}^{\infty} \frac{y(s) x^{\prime}(s) f^{\prime}[x(s)]}{\{f[x(s)]\}^{2}} d s .
$$


We claim that the constant $\theta$ is nonnegative. Otherwise, from $\left(\mathrm{C}_{4}\right)$ and $(\star)$ it follows that there exists $T_{0}^{\star \star} \geq T$ such that

$$
\int_{T_{0}^{\star \star}}^{\infty} \frac{y(s) x^{\prime}(s) f^{\prime}[x(s)]}{\{f[x(s)]\}^{2}} d s \leq-\frac{\theta}{4}
$$

and

$$
\int_{t}^{\infty} a(s) d s \leq-\frac{\theta}{4} \text { for all } t \geq T_{0}^{\star \star} .
$$

Thus, by using ( $\star \star)$, we find for every $t \geq T_{0}^{\star \star}$

$$
\begin{aligned}
-\frac{y\left(T_{0}\right)}{f\left[x\left(T_{0}\right)\right]}+\int_{T_{0}}^{t} a(s) d s+\int_{T_{0}}^{T_{0}^{\star \star}} \frac{y(s) x^{\prime}(s) f^{\prime}[x(s)]}{\{f[x(s)]\}^{2}} d s \\
=-\theta-A\left(T_{0}\right)-\int_{T_{0}}^{\infty} \frac{y(s) x^{\prime}(s) f^{\prime}[x(s)]}{\{f[x(s)]\}^{2}} d s \\
\quad+\int_{T_{0}}^{t} a(s) d s+\int_{T_{0}}^{T_{0}^{\star \star}} \frac{y(s) x^{\prime}(s) f^{\prime}[x(s)]}{\{f[x(s)]\}^{2}} d s \\
=-\theta-\int_{t}^{\infty} a(s) d s-\int_{T_{0}^{\star \star}}^{\infty} \frac{y(s) x^{\prime}(s) f^{\prime}[x(s)]}{\{f[x(s)]\}^{2}} d s \\
\geq-\theta+\frac{\theta}{4}+\frac{\theta}{4}=-\frac{\theta}{2}
\end{aligned}
$$

and so our lemma ensures that

$$
y(t) \leq D \text { for all } t \geq T_{0}^{\star \star},
$$

where $D=(\theta / 2) f\left[x\left(T_{0}^{\star \star}\right)\right]<0$. Hence, exactly as in proving $(\star)$, we arrive at the contradiction $\lim _{t \rightarrow \infty} x(t)=-\infty$, which proves our claim.

Finally, $(\star \star)$ guarantees that

$$
y(t) \geq A(t) f[x(t)] \text { for every } t \geq T_{0} .
$$

Hence, by taking into account the fact that $\lim _{t \rightarrow \infty} A(t)=0$ and using condition $\left(\mathrm{C}_{7}\right)$, from the first equation of $(\mathrm{S})$ we obtain for $t \geq T_{0}$

$$
x^{\prime}(t)=b(t) g[y(t)] \geq b(t) g[A(t) f[x(t)]] \geq b(t) g[A(t)] g\{f[x(t)]\}
$$

and consequently

$$
\int_{x\left(T_{0}\right)}^{x(t)} \frac{d u}{g[f(u)]} \geq \int_{T_{0}}^{t} b(s) g[A(s)] d s \text { for all } t \geq T_{0} .
$$

So, because of condition $\left(\mathrm{C}_{8}\right)$, we have

$$
\int_{T_{0}}^{t} b(s) g[A(s)] d s \leq \int_{x\left(T_{0}\right)}^{\infty} \frac{d u}{g[f(u)]}<\infty \text { for } t \geq T_{0},
$$

which contradicts condition $\left(\mathrm{C}_{9}\right)$. The proof of our theorem is complete. 


\section{REFERENCES}

[1] F. V. Atkinson, On second order nonlinear oscillation, Pacific J. Math. 5 (1955), 643-647. MR 17:264e

[2] S. Belohorec, Oscillatory solutions of certain nonlinear differential equations of second order, Mat. Fyz. Casopis Sloven Akad. Vied. 11 (1961), 250-255. MR 28:3208

[3] W. J. Coles, Oscillation criteria for nonlinear second order equations, Ann. Mat. Pura Appl. 82 (1969), 123-134. MR 41:567

[4] M. K. Kwong and J. S. W. Wong, An application of integral inequality to second order nonlinear oscillation, J. Differential Equations 46 (1982), 63-77. MR 84g:34059

[5] M. K. Kwong and J. S. W. Wong, Oscillation of Emden-Fowler systems, Differential Integral Equations 1 (1988), 133-141. MR 89b:34075

[6] D. D. Mirzov, Oscillatory properties of solutions of a system of nonlinear differential equations, Differentsial'nye Uravneniya 9 (1973), 581-583; English transl., Differential Equations 9 (1973), 447-449. MR 47:3758

[7] D. D. Mirzov, The oscillation of solutions of a system of nonlinear differential equations, Mat. Zametki 16 (1974), 571-576; English transl., Math. Notes 16 (1974), 932-935. MR 51:10762

[8] D. D. Mirzov, Oscillation properties of solutions of a nonlinear Emden-Fowler differential system, Differentsial'nye Uravneniya 16 (1980), 1980-1984; English transl., Differential Equations 16 (1980), 1260-1263. MR 82a:34046

[9] P. Waltman, An oscillation criterion for a nonlinear second order equation, J. Math. Anal. Appl. 10 (1965), 439-441. MR 30:3265

[10] J. S. W. Wong, On two theorems of Waltman, SIAM J. Appl. Math. 14 (1966), 724-728. MR 34:6228

[11] J. S. W. Wong, Oscillation theorems for second order nonlinear differential equations, Proc. Amer. Math. Soc. 106 (1989), 1069-1077. MR 89m:34042

[12] J. S. W. Wong, Oscillation criteria for second order nonlinear differential equations with integrable coefficients, Proc. Amer. Math. Soc. 115 (1992), 389-395. MR 92i:34042

Department of Mathematics, University of Ioannina, 45110 Ioannina, Greece

E-mail address: cphilos@cc.uoi.gr 